\title{
Increasing Hybridization Rate and Sensitivity of Bead-Based Assays Using Isotachophoresis
}

\section{$\operatorname{AUTHOR}(S)$ :}

Shintaku, Hirofumi; Palko, James W.; Sanders, Glenn M.; Santiago, Juan G.

\section{CITATION:}

Shintaku, Hirofumi ...[et al]. Increasing Hybridization Rate and Sensitivity of Bead-Based Assays Using Isotachophoresis. Angewandte Chemie 2014, 126(50): 14033-14036

\section{ISSUE DATE:}

2014-10-10

URL:

http://hdl.handle.net/2433/199656

\section{RIGHT:}

This is the peer reviewed version of the following article: Shintaku, H., Palko, J. W., Sanders, G. M. and Santiago, J. G. (2014), Increasing Hybridization Rate and Sensitivity of Bead-Based Assays Using Isotachophoresis. Angew. Chem., 126: 14033-14036, which has been published in final form at http://dx.doi.org/10.1002/ange.201408403. This article may be used for non-commercial purposes in accordance with Wiley Terms and Conditions for Self-Archiving.; 許諾条件により 本文ファイルは2015-10-10に公開:; This is not the published version. Please cite only the published version.; この論文は 出版社版でありません。引用の際には出版社版をご確認ご利用ください。 


\title{
Increasing hybridization rate and sensitivity of bead based assays using isotachophoresis
}

\author{
Hirofumi Shintaku ${ }^{a, b}$, James W. Palko ${ }^{a}$, Glenn M. Sanders ${ }^{c}$, and Juan G. Santiago ${ }^{a}$ *
}

\begin{abstract}
We present an electrokinetic technique to increase the reaction rate and sensitivity of bead based assays. We use isotachophoresis(ITP) to preconcentrate and co-focus target molecules and beads into a single ITP zone. The process achieves rapid mixing, stirring, and strongly increases binding reaction rate. We demonstrate our assay with quantitative detection of $24 \mathrm{nt}$ single-stranded DNA over a dynamic range of 3 orders of magnitude and multiplexed detection of 10 target species per sample. We show that ITP can achieve approximately the same sensitivity as a well-stirred standard reaction in 60-fold less reaction time (20 min versus $20 \mathrm{~h}$ ). Alternately, compared to standard reaction times of $30 \mathrm{~min}$, we show that $20 \mathrm{~min}$ ITP hybridization can achieve 5.3-fold higher sensitivity.
\end{abstract}

Probe methods such as planar microarrays ${ }^{[1]}$ and bead-based suspension arrays ${ }^{[2]}$ are attractive for molecular diagnostic applications because of their ability to achieve multiplexing and their customizability. The bead suspension format supports lower levels of multiplexing than planar arrays, with approximately several hundred distinguishable bead codes. ${ }^{[2]}$ This level of multiplexing, however, is sufficient for the vast majority of clinical applications $^{[3]}$, and the format offers a number of practical advantages including lower cost than microarrays ${ }^{[4]}$ and high reconfigurability. (New "array" combinations can be achieved by selecting from and mixing beads from a library).

Beads also possess an intrinsic physical advantage to planar substrates in that they distribute the probe molecule throughout the sample volume, thereby reducing the length scale for target transport. Therefore, in some applications, bead assays offer shorter hybridization duration ( $30 \mathrm{~min}$ ) compared to microarrays $(15-24 \mathrm{~h}) .^{[5]}$ Despite this improved mixing, bead assays nevertheless require similar hybridization duration to achieve comparable limits of detection as microarrays. ${ }^{[6]}$ This implies that accelerating the slow reaction of low abundant target remains a challenge.

Isotachophoresis (ITP) is an electrokinetic technique for producing high concentration factors of ionic analytes using a heterogeneous buffer system, composed of a leading electrolyte (LE), and a trailing electrolyte (TE) ${ }^{[7]}$ In peak-mode ITP $^{[8]}$, multiple analytes (e.g., beads and target) sharply focus at a TE-toLE interface resulting in dramatic concentration of analytes (up to million-fold increases are possible within 2 min under ideal conditions ${ }^{[9]}$ ). This rapid mixing, co-focusing, and preconcentration

[a] H. Shintaku, J.W. Palko, J.G. Santiago Department of Mechanical Engineering, Stanford University Stanford, California 94305, United States of America

Email: juan.santiago@stanfod.edu

[b] H. Shintaku

Department of Micro Engineering

Kyoto University

Nishikyo, Kyoto 615-8540, Japan

[c] G.M. Sanders

SomaLogic, Inc.

Boulder, Colorado 80301, United States of America

Supporting information for this article is given via a link at the end of the document. can be used to accelerate second order chemical reactions such as DNA hybridization. ${ }^{[10]}$ Bercovici et al. presented a detailed study of the homogeneous DNA hybridization dynamics under ITP focusing and demonstrated 14000 -fold reduction in hybridization time. ${ }^{[11]}$ Bahga et al. demonstrated a duplex assay by integrating ITP based rapid DNA hybridization and capillary zone electrophoresis. ${ }^{[12]}$ ITP can also enhance the surface hybridization reaction between a suspended target and an immobilized probe. ${ }^{[13]}$ Recently, ITP was used to accelerate selective capture (and recovery) in affinity chromatography ${ }^{[14]}$ and to achieve $30 \mathrm{~min}$ reactions in a multiplexed surface microarray (20 species and 60 reaction spots). ${ }^{[15]}$ The latter work being to our knowledge the first truly multiplexed (e.g., more than two) reactions accelerated using ITP. There have also been studies showing that nano- and microparticles can be focused and carried along with an ITP zone. ${ }^{[16]}$ However, we know of no studies combining ITP-enhanced reactions and bead assays by co-focusing target and beads in an ITP zone.

In this paper, we leverage ITP to concentrate dilute target DNA and co-focus it with beads to strongly accelerate DNA hybridization in a bead suspension assay. Figure 1 shows a schematic of our ITP-aided bead hybridization experiment. We use a simple one-inlet, one-outlet channel architecture and three buffers: LE, a buffering leading electrolyte (BLE), and TE (see S-1 for detailed materials and methods). The LE contained the ssDNA targets and initially filled the entire microchannel. We then injected magnetic bead suspension into the near-entrance region of the microchannel, and collected these into the channel with an external magnet. The bead suspension contained ten sets of beads each with a unique immobilized DNA probe sequence. We then loaded TE and BLE into the input and the output wells, respectively, and placed platinum wire electrodes into the each well.

We applied $300 \mu \mathrm{A}$ of constant current along the channel. ITP focuses all beads within the ITP zone within about 1 min. Target was recruited and focused into the ITP zone and continually accumulated during most of the experiment. Secondary flow in ITP effectively mixes beads and target to achieve accelerated and kinetics-limited reaction (see S-2 of SI for ITP dynamics). We turned off the applied electric field as the ITP zone arrived at the output well and then recovered the solution including processed beads from the output well for off-chip detection of fraction of hybridization.

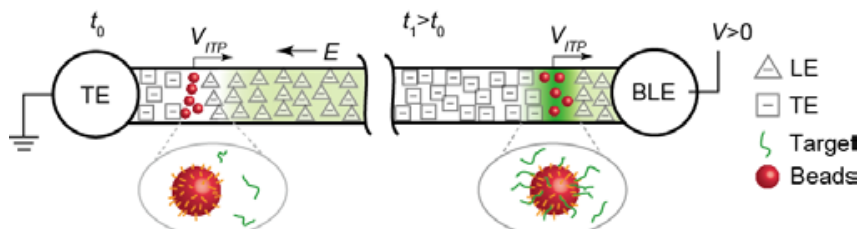

Figure 1 Schematic of hybridization acceleration by co-focusing beads and target in a single ITP zone. Target is initially mixed into LE and distributed throughout channel. ITP focusing quickly accumulates beads injected at the left, and transports focused beads through channel. ITP zone also recruits target, preconcentrates it, and mixes target and beads, accelerating reactions. Beads collected at downstream reservoir with a standard pipette. 
The short, injected bead zone focuses quickly and bead surfaces offer a finite number of and effective volume concentration of possible binding sites. Meanwhile, ITP recruitment of target results in continuous accumulation of target into ITP zone. This recruitment starts as a linear increase in target concentration, $C$, but $C$ saturates as the increasing surface reaction rate rises and becomes sufficient to accommodate new influx, resulting in a quasi-steady local target equilibrium. This quasi equilibrium is a balance between influx of target into ITP zone and reaction rate "sink" within it, and we analyze and quantitatively describe this in the SI (c.f. S-4). The essence of this equilibrium is captured by the following relation:

$$
C^{*}=\alpha \text {. }
$$

Here $C^{*}$ is $C$ normalized by the initial target concentration, $C_{0}$ and $\alpha$ is an acceleration factor determined by ITP chemistry, ITP dynamics, the reaction on-rate constant $\left(k_{o n}\right)$, and the ITP-volumeaveraged concentration of binding sites. Given this quasiequilibrium, we can then describe the fraction of reacted surface sites (normalized by total surface sites), $\Gamma^{*}$, as:

$$
\Gamma^{*}=\alpha /\left(\alpha+K / C_{0}\right)\left[1-\exp \left(-\left(\alpha+K / C_{0}\right) T^{*}\right)\right] \text {, }
$$

where $K$ is the dissociation constant, $T^{*}$ is $k_{o n} C_{0} t$, and $t$ is time. We provide detailed derivation and benchmarking of this ITP-reaction relation in S-5 and S-6. (Refer to S-3 for definition of symbols). We also compare it to an equivalent expression for the well-stirred standard (non-ITP) reaction case, for which the hybridization fraction has the form $\Gamma_{\text {std }}^{*}=1 /\left(1+K / C_{0}\right)\left[1-\exp \left(-\left(1+K / C_{0}\right) T^{*}\right)\right]$.

We here highlight two key differences between ITP and the standard, well-stirred reaction case. First, the ITP hybridization fraction contains a prefactor $\alpha /\left(\alpha+K / C_{0}\right)$. For the common case of low abundance target where $K / C_{0}$ is much larger than unity, the prefactor becomes $\alpha /\left(K / C_{0}\right)$, and we see that ITP focusing can be interpreted as an increased value of steady state target hybridization fraction (the standard fraction multiplied by $\alpha$ ). Second, for the same high $K / C_{0}$ regime, we see that the internal quasi-equilibrium dynamics are associated with a rate of reaction accelerated by a factor $\alpha$. We estimate $\alpha=18.3$ for our ITP experiments (c.f. S-8 of SI).

We experimentally demonstrate our technique by comparing titration curves for ITP and well-stirred standard reactions. We used 10 target-probe pairs composed of commercially available magnetic beads bearing ssDNA probe sequences $(d=6.5 \mu \mathrm{m}$, MagPlex-TAG microspheres, Luminex, Austin, TX), and perfectly complementary target sequences (see S-1 of SI). We present data for ITP, standard $30 \mathrm{~min}$, and standard $20 \mathrm{~h}$ hybridization cases in Figure 2. Here we show representative results for one target (target 8), but include additional results for another target (target 9) in S-9 of SI. The fluorescent bead signal data for these experiments were obtained using a Luminex 200 Instrument (see S-1 of SI). In all cases, the fraction of hybridized probe monotonically increased with increasing concentration over a dynamic range of three orders of magnitude. The fractions of hybridized for $20 \mathrm{~min}$ ITP hybridization were comparable to those of standard hybridization for $20 \mathrm{~h}$ at all concentrations. ITP hybridization yielded significantly higher fractions hybridized compared to standard hybridization for $30 \mathrm{~min}$, showing the acceleration effect of ITP. For example, in the inset, we compare the raw signal for ITP and standard hybridization cases with the lowest target concentration (100 fM) and negative control of no target in solution. The signal relative to the negative control was 10.7 for ITP, 2.82 for standard $30 \mathrm{~min}$, and 11.4 for standard $20 \mathrm{~h}$, showing 5.3 fold sensitivity increase by 20 min ITP compared to standard $30 \mathrm{~min}$.

We also show the fraction of hybridized probes predicted with our analytically-derived Eq. (2) for ITP hybridization and for experimental parameters of $K=7.3 \times 10^{-12} \mathrm{M}, k_{o n}=4.4 \times 10^{6} 1 / \mathrm{Ms}$ and $\Gamma_{\max }=6 \mathrm{nmol} / \mathrm{m}^{2}$ (c.f. SI-7). The approximate analytical model yields good quantitative agreement for the observed trends with no fitting parameter. (Model parameters are determined using separate experiments as described in SI). As a reference, we also show the predicted equilibrium level of fraction of target hybridized, $C^{*}$ eq.

Figure 3 shows measurements of specific and nonspecific signals obtained with ITP, standard hybridization at $30 \mathrm{~min}$, and standard hybridization at $20 \mathrm{~h}$ for target concentrations between $100 \mathrm{fM}$ and $5 \mathrm{nM}$. The signals represent the averaged median fluorescence intensity (MFI) resulting from specific binding of ssDNA to the matched probe (ID $=8$ ) or nonspecific binding of ssDNA to the mismatched probes (others). Figure 3b further shows plots of the so-called specificity index, ${ }^{[15]}$ defined as the ratio of specific signal versus the highest non-specific signal (ID $=4$ for the case of target 8). (No data is shown for $100 \mathrm{fM}$ as there was no detectable nonspecific signal above background.) The specificity index from ITP and standard $20 \mathrm{~h}$ incubation were the same order of magnitude and show similar trends. ITP yields higher specificity index than standard incubation for target 8 concentrations below $10 \mathrm{pM}$. We present additional specificity data for target 9 in S-9 in SI which shows ITP can also have slightly lower specificity index at low concentrations. Overall, we concluded that ITP exhibits specificity on par with standard hybridizations. We note Han et al. reached a similar conclusion regarding specificity for their ITPaided DNA microarray hybridizations. ${ }^{[15]}$

In summary, we demonstrated the use of ITP to co-focus, mix, and react nucleic acid targets and multiplexed assay beads in suspension. ITP hybridizations lasting only $20 \mathrm{~min}$ produce sensitivities similar to $20 \mathrm{~h}$ of well-stirred standard hybridizations. 20 min ITP hybridization yields a 5 fold enhancement in sensitivity compared to standard assay protocols with 30 min hybridization. ITP-based hybridization has potential to dramatically speed up bead hybridizations while also improving their sensitivity.

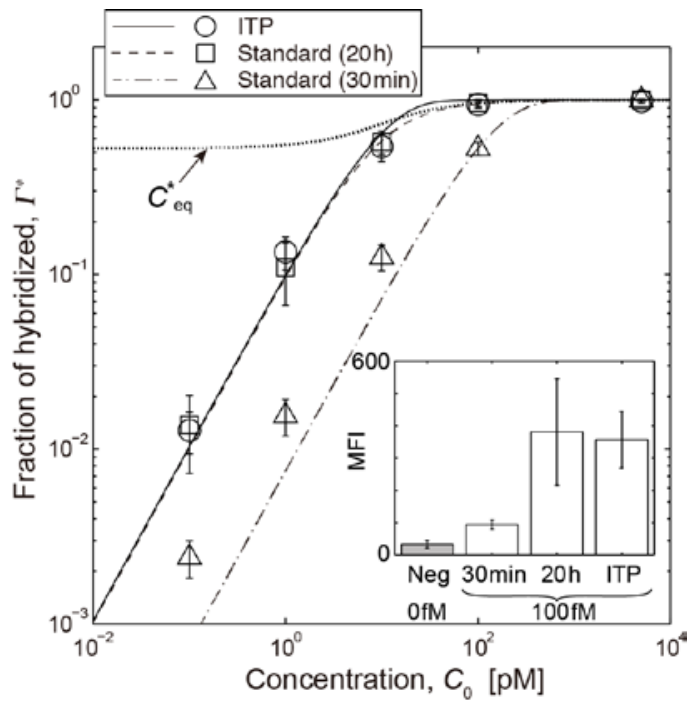

Figure 2 Experimental data demonstrating quantitative detection of a target molecule using 20 min bead-based ITP hybridization and its comparison to well-stirred standard hybridizations performed for $30 \mathrm{~min}$ and $20 \mathrm{~h}$. We obtained the titration curves for target 8 concentraton ranging from $100 \mathrm{fM}$ to $5 \mathrm{nM}$. Along with experimental data (symbols), we show prediction of the analytical model for ITP hybridization (solid) with no fitting parameter. We also show results of analytical models for the standard $20 \mathrm{~h}$ (broken) and $30 \mathrm{~min}$ (dashed-dotted) hybridizations. The range bars in the figure indicate the standard deviation across repetitiation $(N=6$ for standard or $N=9$ for ITP). The dotted line shows the ideal equilibirum target concentration at standard hybridization conditions. The inset shows 
raw signals at an initial target concentration of $100 \mathrm{fM}$ and compares these to the negative control (no target).

\section{Acknowledgements}

We gratefully acknowledge Mr. Y. Sato and Mr. N. Hatano, Kyoto University Machine Shop, for fabricating microfluidic chips. We gratefully acknowledge funding from the National Science Foundation under CBET-1159092. H.S. acknowledges funding from JSPS under 22686021, 26289035, and 26630052. H.S. was supported by fellowships from the John Mung Program of Kyoto University and Marubun Research Promotion Foundation, Japan.

Keywords: isotachophoresis $\bullet$ bead-based assay $\bullet$ multiplexing - DNA analysis $\bullet$ hybridization assay

[1] C. G. Liu, G. A. Calin, S. Volinia, C. M. Croce, Nat Protoc 2008, 3, 563-578

[2] H. Y. Hsu, T. O. Joos, H. Koga, Electrophoresis 2009, 30 4008-4019.

[3] H. S. Eng, G. Bennett, P. Bardy, P. Coghlan, G. R. Russ, P. T. Coates, Hum Immunol 2009, 70, 595-599.

[4] J. Q. Yin, R. C. Zhao, K. V. Morris, Trends Biotechnol 2008, 26, 70-76.

[5] M. Schmitt, I. G. Bravo, P. J. Snijders, L. Gissmann, M. Pawlita, T. Waterboer, J Clin Microbiol 2006, 44, 504-512.

[6] a) J. Lu, G. Getz, E. A. Miska, E. Alvarez-Saavedra, J. Lamb, D. Peck, A. Sweet-Cordero, B. L. Ebert, R. H. Mak, A. A. Ferrando, J. R. Downing, T. Jacks, H. R. Horvitz, T. R. Golub, Nature 2005, 435, 834-838; b) S. Kraemer, J. D. Vaught, C. Bock, L. Gold, E. Katilius, T. R. Keeney, N. Kim, N. A. Saccomano, S. K. Wilcox, D. Zichi, G. M. Sanders, PLoS One 2011, 6, e26332.

a) A. Rogacs, L. A. Marshall, J. G. Santiago, J Chromatogr A 2014, 1335, 105-120; b) H. Shintaku, H. Nishikii, L. A. Marshall, H. Kotera, J. G. Santiago, Anal Chem 2014, 86, $1953-$ 1957.

[8] T. K. Khurana, J. G. Santiago, Anal Chem 2008, 80, 6300-6307.

[9] B. Jung, R. Bharadwaj, J. G. Santiago, Anal Chem 2006, 78, 2319-2327.

[10] a) G. Goet, T. Baier, S. Hardt, Lab Chip 2009, 9, 3586-3593; b) A. Persat, J. G. Santiago, Anal Chem 2011, 83, 2310-2316; c) M. Bercovici, G. V. Kaigala, K. E. Mach, C. M. Han, J. C. Liao, J. G. Santiago, Anal Chem 2011, 83, 4110-4117; d) C. Eid, G. Garcia-Schwarz, J. G. Santiago, Analyst 2013, 138, 3117-3120.

[11] M. Bercovici, C. M. Han, J. C. Liao, J. G. Santiago, Proc Natl Acad Sci USA 2012, 109, 11127-11132.

[12] S. S. Bahga, C. M. Han, J. G. Santiago, Analyst 2013, 138, 8790.

[13] a) G. Garcia-Schwarz, J. G. Santiago, Anal Chem 2012, 84, 6366-6369; b) G. Garcia-Schwarz, J. G. Santiago, Angew Chem Int Ed Engl 2013, 52, 11534-11537; c) M. Karsenty, S. Rubin, M. Bercovici, Anal Chem 2014, 86, 3028-3036; d) R. Khnouf, G. Goet, T. Baier, S. Hardt, Analyst 2014, 139, 4564-4571.

[14] V. Shkolnikov, J. G. Santiago, Anal Chem 2014, 86, 6229-6236.

[15] C. M. Han, E. Katilius, J. G. Santiago, Lab Chip 2014, 14, $2958-$ 2967.
[16] a) U. Pyell, W. Bucking, C. Huhn, B. Herrmann, A. Merkoulov, J. Mannhardt, H. Jungclas, T. Nann, Anal Bioanal Chem 2009 395, 1681-1691; b) G. Goet, T. Baier, S. Hardt, Biomicrofluidics 2011, 5, 014109 .

a
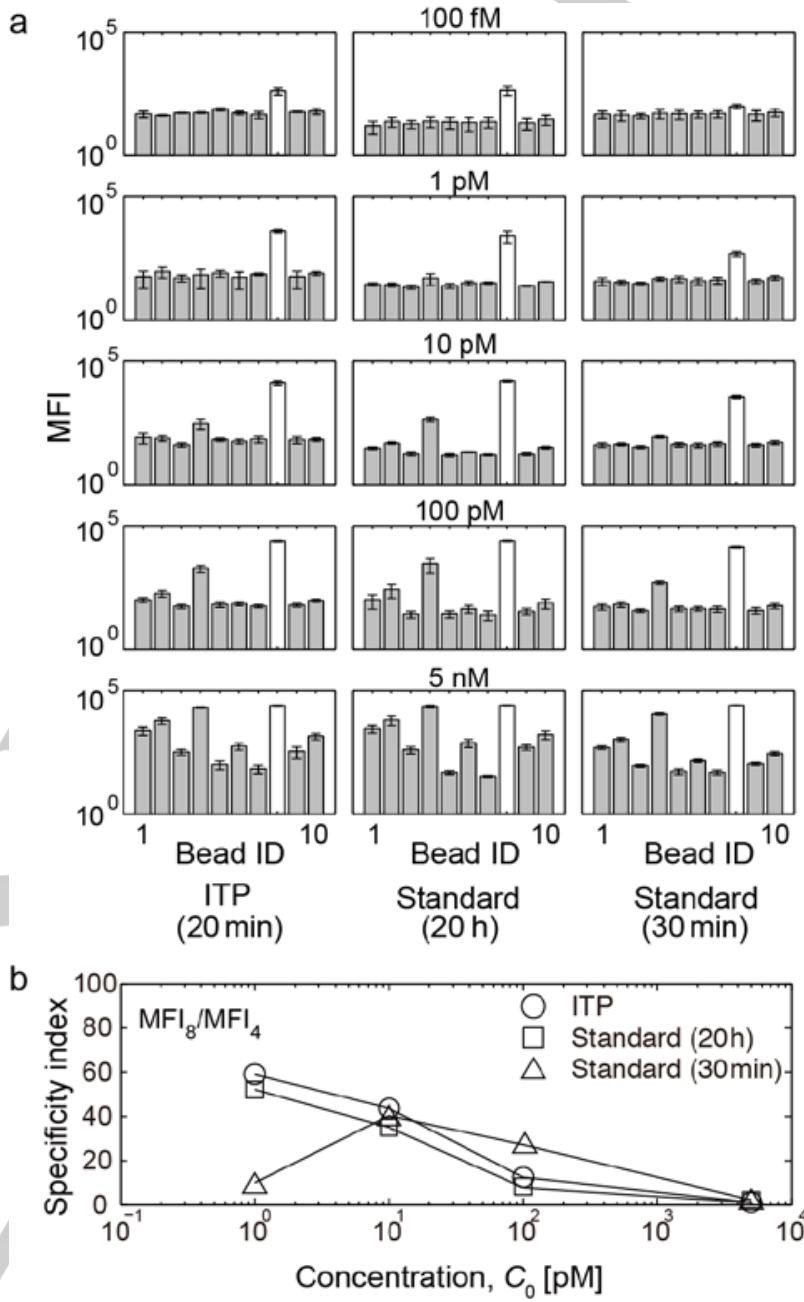

Figure 3 Comparison of specific and nonspecific signal between ITP hybridization and standard incubation (at both $20 \mathrm{~h}$ and $30 \mathrm{~min}$ ). a) Averaged MFI (log scale) for target 8 hybridized with matching probe (bead ID=8) and nine bead types with non-matching probes. The range bars in the figure indicate the standard deviation across $N=3$ for standard cases and $N=5$ for ITP. b): Specificity index for target 8 (ratio of averaged MFI between specific binding and the highest nonspecific binding (bead ID=4). Similar data is provided in the SI for target 9 . 


\section{Entry for the Table of Contents}

\section{COMMUNICATION}

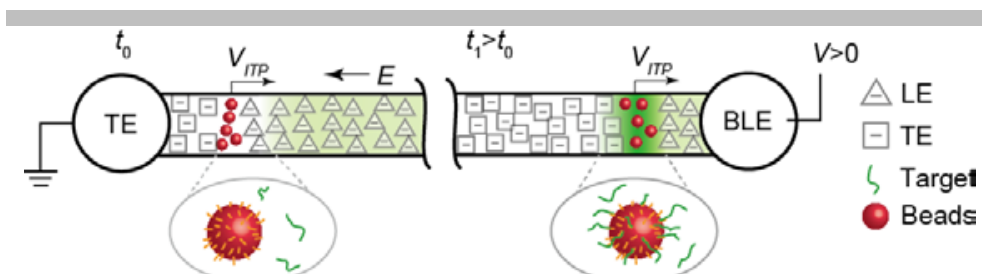

A bead-based assay that uses electrokinetic co-focusing of target molecules and beads to speed up reaction. This technique achieves quantification of ssDNA with $100 \mathrm{fM}$ limit of detection and multiplex detection of 10 target species per sample. The technique offers similar sensitivity to a well-stirred standard reaction in 60-fold less reaction time. Alternately, for similar reaction time, it offers 5.3-fold higher sensitivity.
H. Shintaku, J.W. Palko, G.M. Sanders, J.G. Santiago*

Page No. - Page No.

Increasing hybridization rate and sensitivity of bead based assays using isotachophoresis 
Supporting Information

\title{
Increasing hybridization rate and sensitivity of bead based assays using isotachophoresis
}

\author{
Hirofumi Shintaku ${ }^{\mathrm{a}, \mathrm{b}}$, James W. Palko ${ }^{\mathrm{a}}$, Glenn M. Sanders ${ }^{\mathrm{c}}$, and Juan G. Santiago ${ }^{\mathrm{a}^{*}}$ \\ ${ }^{a}$ Department of Mechanical Engineering, Stanford University, United States of America \\ ${ }^{b}$ Department of Micro Engineering, Kyoto University, Japan \\ 'SomaLogic, Inc., United States of America \\ * To whom correspondence should be addressed.E-mail: juan.santiago@stanford.edu \\ Contents:

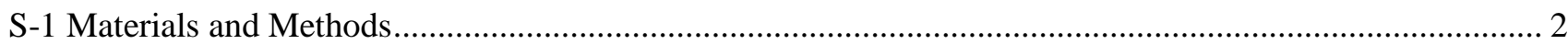

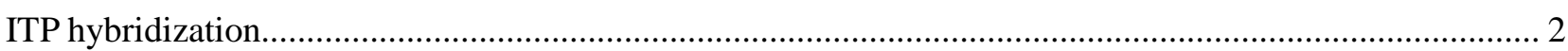

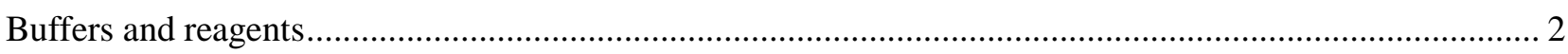

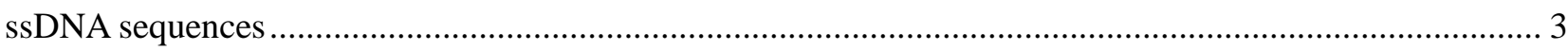

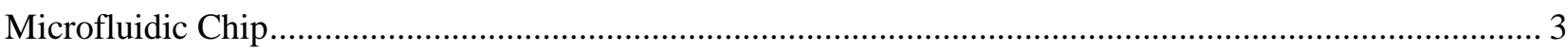

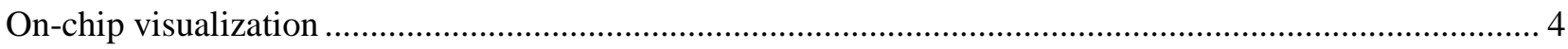

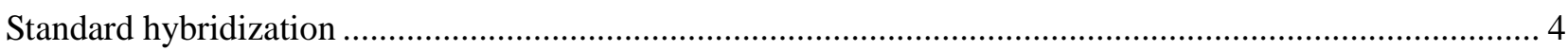 \\ Off-chip Luminex bead signal readout.................................................................................................... 4
}

S-2 Visualization of co-focusing of beads and ssDNA in ITP zone ............................................................... 5

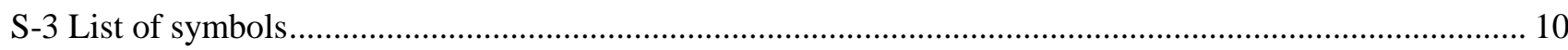

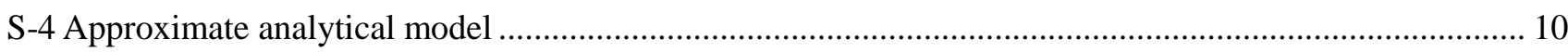

S-5 Benchmarking approximate analytical solution with numerical model.................................................. 12

S-6 Theory for standard incubation hybridization and process of estimating dissociation constant and kinetic on-rate

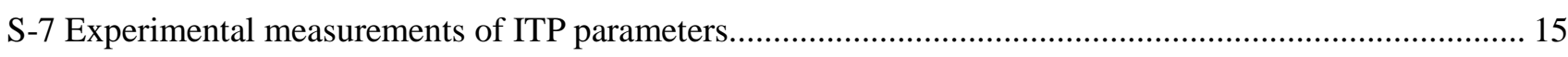

S-8 Predicted gains by approximate analytical model in the fraction of hybridized probes using ITP............. 16

S-9 Example data from additional titration and specificity experiments ..................................................... 18

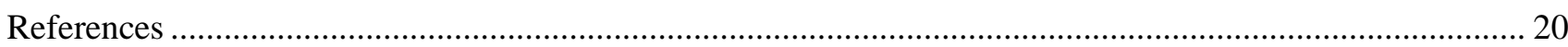




\section{S-1 Materials and Methods}

\section{ITP hybridization}

Our ITP hybridization uses a simple one-inlet, one-outlet channel architecture and three buffers: LE, BLE, and TE. The LE contained the ssDNA targets and initially filled the entire microchannel ( $25 \mu \mathrm{L}$ ) by capillary force. We then injected $0.5 \mu \mathrm{L}$ of magnetic bead suspension at the entrance of the microchannel. The bead suspension contained ten sets of beads each with a different probe sequence and approximately $1,450 \pm 70$ beads in total (145 beads per probe sequence). In this injection step, we dragged the beads into the channel with a permanent magnet placed below the microfluidic chip near the entrance. We then loaded $80 \mu \mathrm{L}$ of TE and BLE into the input and the output wells, respectively, and placed platinum wire electrodes into the each well.

We initiated ITP by applying $300 \mu \mathrm{A}$ of constant current across the channel with input well grounded using a high voltage sourcemeter (2410, Keithley Instruments, Cleveland, OH). Under the action of the electric field, beads were focused in the ITP zone, and target was continually accumulated in the zone as it swept through the channel (see S-2 in SI for ITP dynamics). We turned off the applied electric field as the ITP zone arrived at the output well and then recovered the solution including processed beads from the output well. After the ITP hybridization experiment, we recovered the beads from the output well with a micro-pipette. We estimated the recovery efficiency of beads including beads lost in the pipetting step as $75 \pm 4 \%$ (S.E., $N=5$ ).

During ITP, we applied a rotational magnetic field using a magnetic stirrer (Thermix Stirrer Model 120S, Thermo Fisher Scientific, Waltham, MA) to reduce both bead aggregation and adhesion to the wall. The ITP was very repeatable, and the assay time was $13,520 \pm 60 \mathrm{~s}$.

\section{Buffers and reagents}

For the hybridization experiments, we used 10 target-probe pairs composed of commercially available magnetic beads bearing ssDNA probe sequences $(d=6.5 \mu \mathrm{m}$, MagPlex-TAG microspheres, Luminex, Austin, TX), and perfectly complementary target sequences. We provide sequences of target and probe DNA oligonucleotides in the next section. The target sequences were synthesized with a biotin moiety at the 5' terminus. We arbitrarily named target-probe pairs with ID numbers from 1 to 10. Targets and probes had the same length, 24 bases. We purchased targets from Trilink (San Diego, CA). We also used ssDNA oligo target labeled with Alexa Fluor 488 (AF488, Integrated DNA Technologies, Coralville, IA) at 5' terminus for on-chip visualization and for experimental measurement of capture probe surface concentration, $\Gamma_{\max }$.

For ITP hybridization, the aqueous buffer inside the channel, designated LE, contained $150 \mathrm{mM}$ $\mathrm{HCl}, 300 \mathrm{mM}$ Bis-Tris, $5 \mathrm{mM} \mathrm{MgCl} 2,0.2 \%$ w/w $1 \mathrm{MDa}$ poly(vinylpyrrolidone) (PVP), 0.08\% w/w Triton X-100, and varying concentrations of ssDNA target mixtures. The buffer in the output well, 
BLE, was $250 \mathrm{mM} \mathrm{HCl}, 500 \mathrm{mM}$ BisTris, $5 \mathrm{mM} \mathrm{MgCl}$, 0.2\% w/w $1 \mathrm{MDa}$ poly(vinylpyrrolidone) (PVP), and $0.08 \% \mathrm{w} / \mathrm{w}$ Triton X-100. The trailing electrolyte buffer, TE, contained $250 \mathrm{mM}$ HEPES, $500 \mathrm{mM}$ Bis-Tris, 0.2\% w/w PVP, and 0.08\% w/w Triton X-100. For conventional hybridization, we mixed target DNA in LE buffer to final concentrations ranging from $100 \mathrm{fM}$ to $5 \mathrm{nM}$.

We purchased $\mathrm{HCl}$, HEPES, Tris, Bis-Tris, $\mathrm{MgCl}_{2}$, Triton X-100 from Sigma-Aldrich (St. Louis, MO), and PVP from Polysciences, Inc. (Warrington, PA). We prepared all solutions in UltraPure DNase free distilled water (GIBCO Invitrogen, Carlsbad, CA).

\section{SSDNA sequences}

We used ssDNA sequences for targets and probes as listed in Table S1. Targets include a biotin group at the 5' terminus. We calculated melting temperatures $T_{\mathrm{m}}$ of each target-probe pair using the DINAMelt web server (http://mfold.rna.albany.edu/). This estimate took into account the effect of ionic concentration by using the dissociated Bis-Tris concentration, $150 \mathrm{mM}$, and substituting this value for the input $\mathrm{Na}^{+}$concentration required by the calculation.

Table S1. Probe and target oligonucleotide sequences

\begin{tabular}{ccccc}
\hline ID & Name & Tag sequence $\left(5^{\prime} \rightarrow 3^{\prime}\right)$ & Anti-tag sequence $\left(5^{\prime} \rightarrow 3^{\prime}\right)$ & $T_{\mathrm{m}}$ \\
\hline 1 & MTAG-A012 & AGTAGAAAGTTGAAATTGATTATG & CATAATCAATTTCAACTTTCTACT & 65.8 \\
2 & MTAG-A013 & AGTGAATGTAAGATTATGTATTTG & CAAATACATAATCTTACATTCACT & 64.9 \\
3 & MTAG-A014 & ATTGTGAAAGAAAGAGAAGAAATT & AATTTCTTCTCTTTCTTTCACAAT & 68.4 \\
4 & MTAG-A015 & GTTGTAAATTGTAGTAAAGAAGTA & TACTTCTTTACTACAATTTACAAC & 64.6 \\
5 & MTAG-A019 & GTGTGTTATTTGTTTGTAAAGTAT & ATACTTTACAAACAAATAACACAC & 65.6 \\
6 & MTAG-A025 & GTATGTTGTAATGTATTAAGAAAG & CTTTCTTAATACATTACAACATAC & 63.3 \\
7 & MTAG-A026 & TTTGATTTAAGAGTGTTGAATGTA & TACATTCAACACTCTTAAATCAAA & 67.9 \\
8 & MTAG-A027 & AAGATGATAGTTAAGTGTAAGTTA & TAACTTACACTTAACTATCATCTT & 67.2 \\
9 & MTAG-A030 & GTGTTATAGAAGTTAAATGTTAAG & CTTAACATTTAACTTCTATAACAC & 62.9 \\
\hline 10 & MTAG-A055 & GAAGATATTGAAAGAATTTGATGT & ACATCAAATTCTTTCAATATCTTC & 68.0 \\
\hline
\end{tabular}

\section{Microfluidic chip}

The microfluidic chip design had a nominal channel depth of $130 \mu \mathrm{m}$, a nominal width of $2 \mathrm{~mm}$, and a nominal total channel length of $99 \mathrm{~mm}$ as shown in Fig. S1. Each channel corner is an optimized $90^{\circ}$ turn to minimize electrokinetic dispersion. ${ }^{[1]}$ The channel contained approximately $25 \mu \mathrm{L}$. At both ends, the microchannel had wells that hold a total volume of $80 \mu \mathrm{L}$.

We fabricated the microfluidic channel structure on a 2 mm-thick acrylic plate using 
conventional computer numerically controlled milling. We cleaned the plate in DI water with sonication for $5 \mathrm{~min}$. We sealed the channel with another $500 \mu \mathrm{m}$-thick acrylic plate by compression at $105^{\circ} \mathrm{C}$ for $30 \mathrm{~min}$.

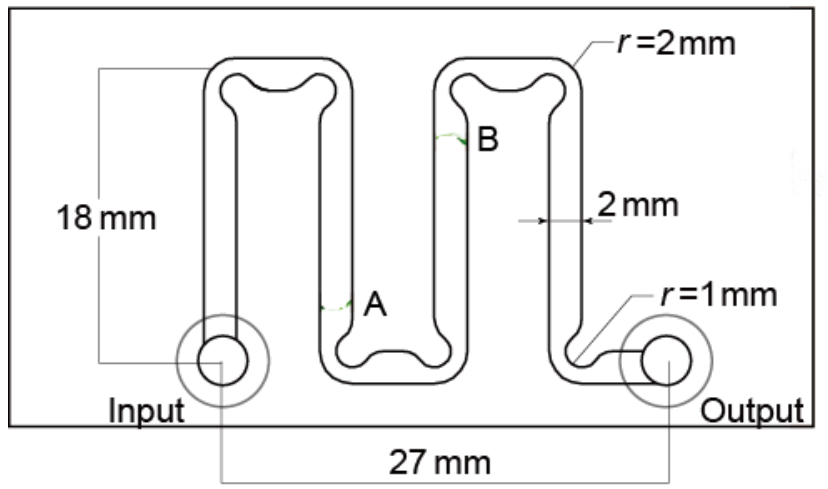

Figure S 1 Schematic of the microchannel.

\section{On-chip visualization}

For on-chip visualization of bead hybridization, we used an inverted epifluorescence microscope (IX70, Olympus, Tokyo, Japan) equipped with a 4X objective lens (UPlanApo, NA 0.16, Olympus, Tokyo, Japan) and a 0.63X demagnification lens (Diagnostic Instruments, Sterling Heights, MI). To individually and simultaneously observe beads versus targets, we captured simultaneous images in two separate wavelength bands using an XF53 dual pass filter cube (Omega Optical, Brattleboro, VT) in combination with a dual-view imager (Micro-Imager, Photometrics, Tucson, AZ). We captured images using a CCD camera (RTE/CCD-1300-Y/DIF, Roper Scientific, Trenton, NJ) with $2 \times 2$ binning. We controlled the camera under double image feature mode and external exposure control with an exposure time of 125 ms.

\section{Standard hybridization}

For standard hybridization experiments, we prepared $50 \mu \mathrm{L}$ bead suspensions including $5810 \pm 290$ beads $(N=3)$ in LE and mixed with $50 \mu \mathrm{L}$ of target ssDNA solution in a $0.5 \mathrm{~mL}$ microtube. We then incubated the mixed solution on a hotplate at $37^{\circ} \mathrm{C}$ with stirring at $70 \mathrm{RPM}$ by a magnetic hot stirrer (Isotemp, Fisher Scientific, Pittsburgh, PA). For these conventional comparison cases, we used two incubation times: $20 \mathrm{~h}$ and $30 \mathrm{~min}$. The assay with $20 \mathrm{~h}$ incubation provides results near equilibrium and offers high sensitivity. Multiple assays performed with 30 min incubation, which Luminex standard protocol recommends, enables a measurement of $k_{o n}$.

\section{Off-chip Luminex bead signal readout}

Following both microfluidic ITP and standard hybridizations, we used a protocol and bead signal readout method common to Luminex bead signal analysis. We performed this in order to have a fair comparison of both hybridization methods. Also, we believe our assay's main contribution is in 
replacing the mixing and incubation/hybridization reaction steps of the accepted overall assay. To this end, we performed the following off-chip sample preparation and quantification in the Human Immune Monitoring Center at Stanford University. We loaded each processed bead suspension into separate wells of a 96 well plate (655096, Greiner Bio-One, NC, USA). We washed beads with phosphate buffered saline solution (PBS) including 0.05\% Tween 20 (PBST) in an automated washer (ELx405, Biotek, VT, USA). We then suspended the beads in a buffer $(0.2 \mathrm{M} \mathrm{NaCl}, 0.1 \mathrm{M}$ Tris, and $0.08 \%$ Triton $\mathrm{X}-100$ at $\mathrm{pH} 8.0$ ) including $0.5 \mathrm{mg} / \mathrm{mL}$ bovine serum albumin (Invitrogen, Carlsbad, CA) for 5 min with shaking. We placed the plate on a magnetic separator for 5 min and removed the supernatant. We then added a buffer $(0.2 \mathrm{M} \mathrm{NaCl}, 0.1 \mathrm{M}$ Tris, and $0.08 \%$ Triton $\mathrm{X}-100$ at $\mathrm{pH}$ 8.0) including $5 \mu \mathrm{g} / \mathrm{mL}$ fluorophore of streptavidin-R-phycoerythrin (SAPE) and incubated at room temperature for 40 min with shaking. We washed beads with PBST in a Biotek ELx405 washer and re-suspended them in PBS for reading with the Luminex 200 Instrument (Luminex, Austin, TX). This standard post-reaction protocol with standard equipment resulted in the most significant loss of reacted beads. In all, the Luminex 200 Instrument quantified about 20 beads per type. We used median fluorescence intensity (MFI) as the representative signal. We estimated the fraction of hybridized probe by normalizing the negative-control-subtracted fluorescence signal with the maximum value.

\section{S-2 Visualization of co-focusing of beads and ssDNA in ITP zone}

We performed on-chip visualization experiments of the ITP hybridization using ssDNA labeled with AF488. For these hybridization visualizations, we focused beads and ssDNA at $10 \mathrm{nM}$ initial concentration. Our optimized ITP chemistry allows co-focusing of beads and ssDNA in a single ITP zone. During focusing, we leverage the strong secondary flow in the ITP zone to mix and achieve kinetically limited reaction by applying relatively high current. We provide example visualizations (and velocity measurements) of this random, fluctuating secondary flow in Fig. S2 and within the multimedia SI. We also observe spanwise non-uniform distribution of target and beads (vertical direction in Fig. S2). The secondary flow and non-uniformity in the spanwise distribution became stronger as the ITP zone moved to the output well under our constant current condition. We attribute this to the effects of increasing electric field (as more of the channel is occupied by lower conductivity TE zone). As we describe below, increasing electric fields cause stronger secondary flows associated with non-uniform electroosmotic flow (EOF) and electrokinetic flow instabilities.

For negligible electroosmotic flow (EOF), low electric fields, and peak mode, ${ }^{[2]}$ ITP zone shape is well predicted by a balance of electrophoretic focusing gradients and molecular diffusion. ${ }^{\text {[3] }}$ The ITP zone can exhibit "tails" into the TE and LE zones for cases where the focused species 
mobility approaches that of the TE or LE co-ions, respectively. ${ }^{[3]}$ Finite electroosmotic flow can affect ITP zone shape by causing Taylor-type dispersion near the boundaries between the ITP zone peak and the TE and LE. At higher electric fields, we have observed distortion of ITP zone shape, which we attribute to both non-uniform electroosmotic flow and the effects of electric body forces. ${ }^{\text {[3] }}$ At sufficiently high electric fields, we observe three-dimensional fluctuations of analyte concentrations which we attribute to electrokinetic flow instabilities. ${ }^{[4]}$ The non-dimensional parameters governing this transition to instability are not well known precisely but Persat and Santiago present scaling arguments suggesting a modified electric Rayleigh number. ${ }^{[4]}$

Generally speaking, the result of these complex effects on ITP zone shape can be summarized as follows. For relatively low electric fields and suppressed EOF, we can assume ITP zone width will be roughly inversely proportional to current density. ${ }^{[3]}$ At higher values, the dispersive action of secondary flows and instabilities tends to produce a plateau minimum ITP zone width and strongly fluctuating scalar flows and mixing within the ITP zone. As part of the current work, we leveraged these instabilities and secondary flows to effect strong mixing between focused beads and focused target molecules within the ITP zone. We here present instantaneous scalar images of the ITP zone and nearly instantaneous velocity measurements using particle image velocimetry.

First, we present scalar images visualized with 24 nt ssDNA end-labeled with AF488 in Fig.S2. The images in Figs. S2a and b are from a single experiment, recorded at different straight channel regions indicated as A and B, respectively, in Fig.S1. In Figs. S2a and b, the green color is $24 \mathrm{nt}$ ssDNA end-labeled with AF488, and the pink dots (black dots in ITP zone) are beads. The spatial distribution of the scalar is relatively stable within the straight region, while it dramatically changes at turns (data not shown). The scalar shows span-wise non-uniform distribution, and tends to accumulate at the outer edge of the channel, which are the top and the bottom edges in Figs. S2a and b, respectively. Paschkewitz et al. also reported the turn induced outer side scalar accumulation. ${ }^{\text {[5] }}$ Figures S2a and b also show the co-focused beads as pink dots (black dots in the ITP zone). We observed that the spatial distribution of beads is also relatively stable within straight regions, while individual beads dynamically move in the ITP zone.

Next, we characterized the secondary flow velocities within the ITP zone using particle image velocimetry as shown in Fig.S2c. We observed rotational motion of beads in the ITP zone with the velocity order of $10 \mu \mathrm{m} / \mathrm{s}$, which was one order of magnitude smaller than that of ITP zone translational migration. To obtain the velocity field in the frame of reference of the moving ITP interface, we recorded the visualized ITP zone, manually tracking the ITP zone by moving the microscope stage. For more precise registration of multiple images of the ITP zone, we used the green channel fluorescence signal image representing the diffuse target signal to track the ITP interface with the two-dimensional cross-correlation 'xcorr2' MATLAB function. This analysis 
yielded the ITP translational shift distances by which we then shifted the red fluorescence channel images. We analyzed the discrete position of the red fluorescent beads in the frame of reference of the moving ITP zone (as determined by the green channel). We used the preconditioned red channel images to analyze the beads' motion in the ITP zone by micron-resolution particle image velocimetry. ${ }^{[6]}$ We used 32 by 32 pixel interrogation windows with $50 \%$ overlap. As the structure of secondary flows were approximately steady for time scales of order $100 \mathrm{~s}$ and within the straight region of the microchannel (in the moving frame of reference), we averaged velocity information within the straight region by ensemble averaging 44 correlation functions (each associated with an image pair) per velocity calculation. ${ }^{[7]}$ For the experimental data in Fig. S2c, we also show the averaged green channel image intensity in grayscale to indicate the ITP zone.

We also measured $\delta$ as an "effective" ITP zone length. In Fig. S3 we present the ITP zone length (along channel axis) versus peak position. We observed the ITP zone length increased with the migration of the ITP zone. Our experiments were run at constant current of $300 \mu \mathrm{A}$, and this results in an increasing electric field within the TE zone. As mentioned above, the TE zone has lower conductivity than the LE and progressively occupies more of the channel (resulting in increasing EOF within the TE zone). ${ }^{[3]}$ We also measured $\delta$ at constant current of $200 \mu \mathrm{A}$ and observed $\delta$ was relatively insensitive to the current in our dispersive regime. 
a

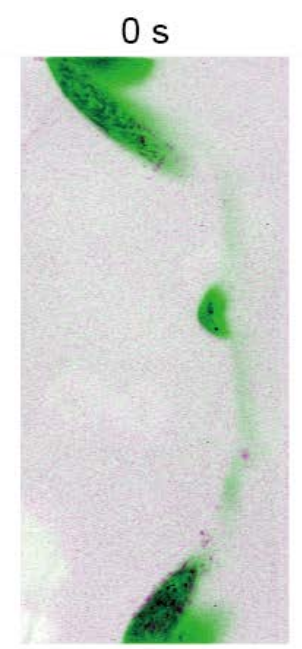

Migration directoin
$10 \mathrm{~s}$

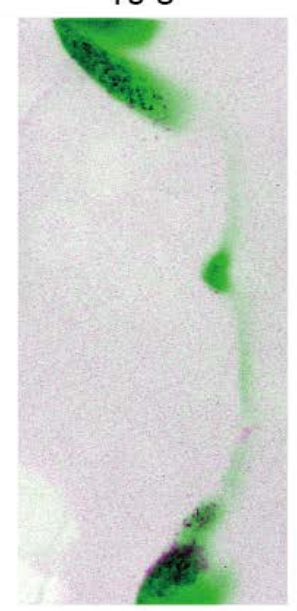

$10 \mathrm{~s}$

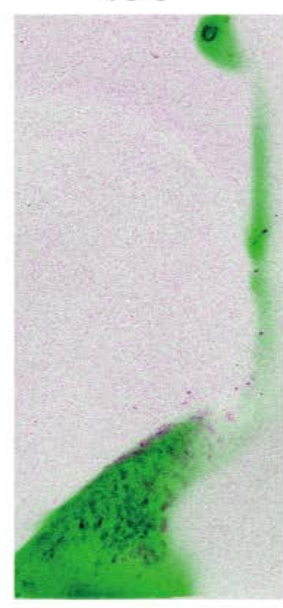

Migration directoin

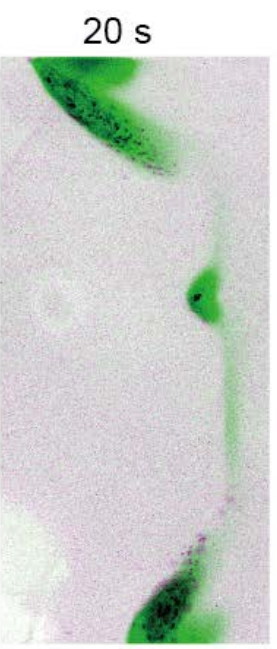

$20 \mathrm{~s}$
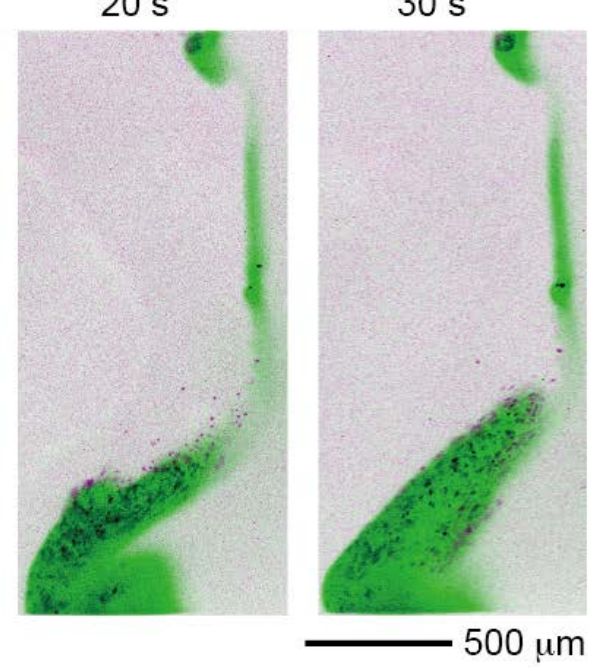

$30 \mathrm{~s}$

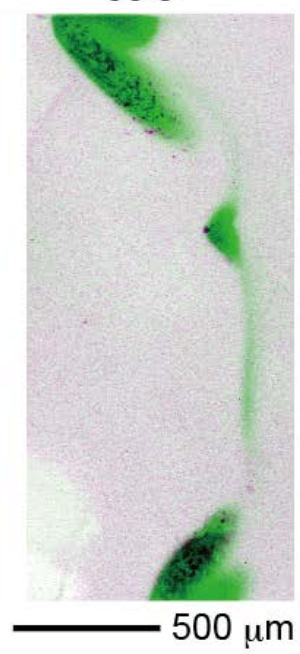

C

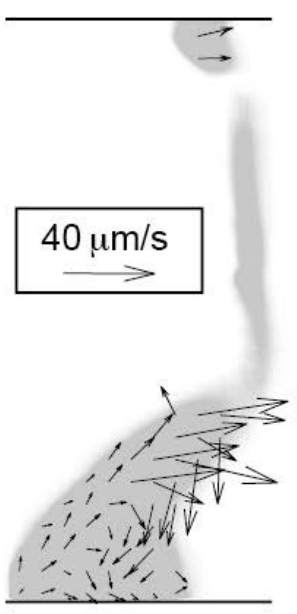

Figure S 2 Experimental demonstration of mixing action of ITP during co-focusing of beads and target molecule in a single ITP zone. The initial target concentration used solely for this example visualization was $10 \mathrm{nM}$. We recorded image sequences a) and b), respectively, in the straight zones A and B indicated in Fig.S1. Here, the green color is 24 nt ssDNA end-labeled with AF488, and the pink dots (black dots in ITP zone) are beads. c) An example of experimentally measured bead motion due to secondary flow in ITP zone. The strong secondary flow in ITP zone effectively mixes beads and target to achieve kinetically limited reaction and resulted in a uniform reaction over all the beads. 


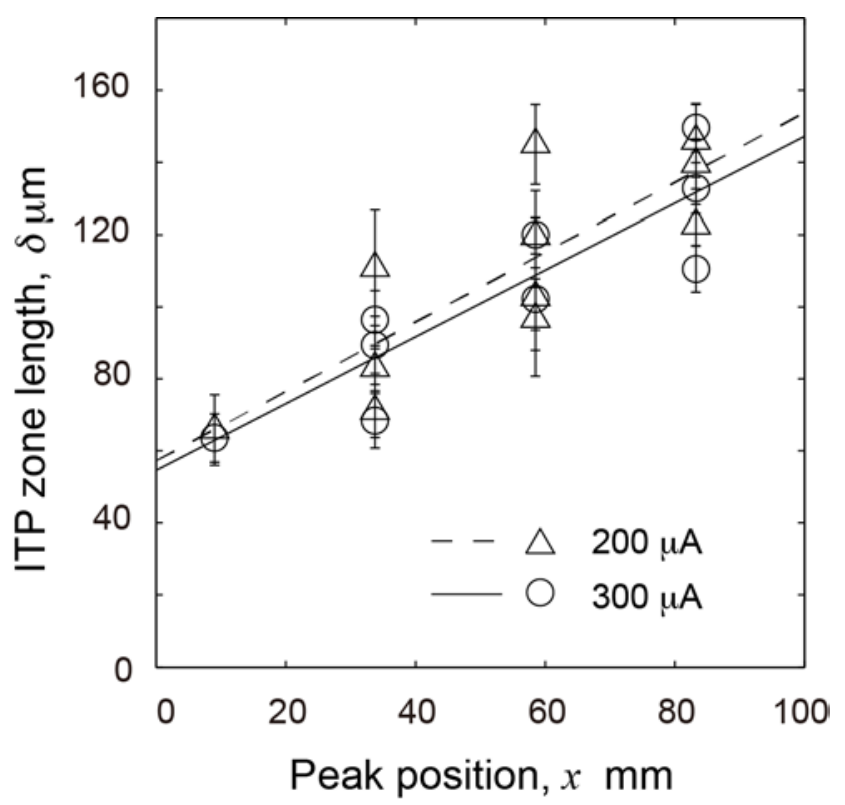

Figure S 3 Measurements of ITP zone length as a function of ITP zone position with applied constant currents of $200 \mu \mathrm{A}$ (triangles, broken line) and $300 \mu \mathrm{A}$ (circles, solid line). Each symbol shows the averaged ITP zone length at indicated positions along the $18 \mathrm{~mm}$ long straight zone of the microchannel, where we used the central position, $x$, as a representative peak position. For this estimate, we first determined the ITP zone as the region where the fluorescence intensity from the target molecule is higher than a threshold, $I_{\mathrm{bk}}+\sigma . \quad I_{\mathrm{bk}}$ is the mean intensity of the background fluorescence within the channel and $\sigma$ is the standard deviation of this background. We then measured the imaged area of the ITP zone and divided by the width of channel to obtain $\delta$. 


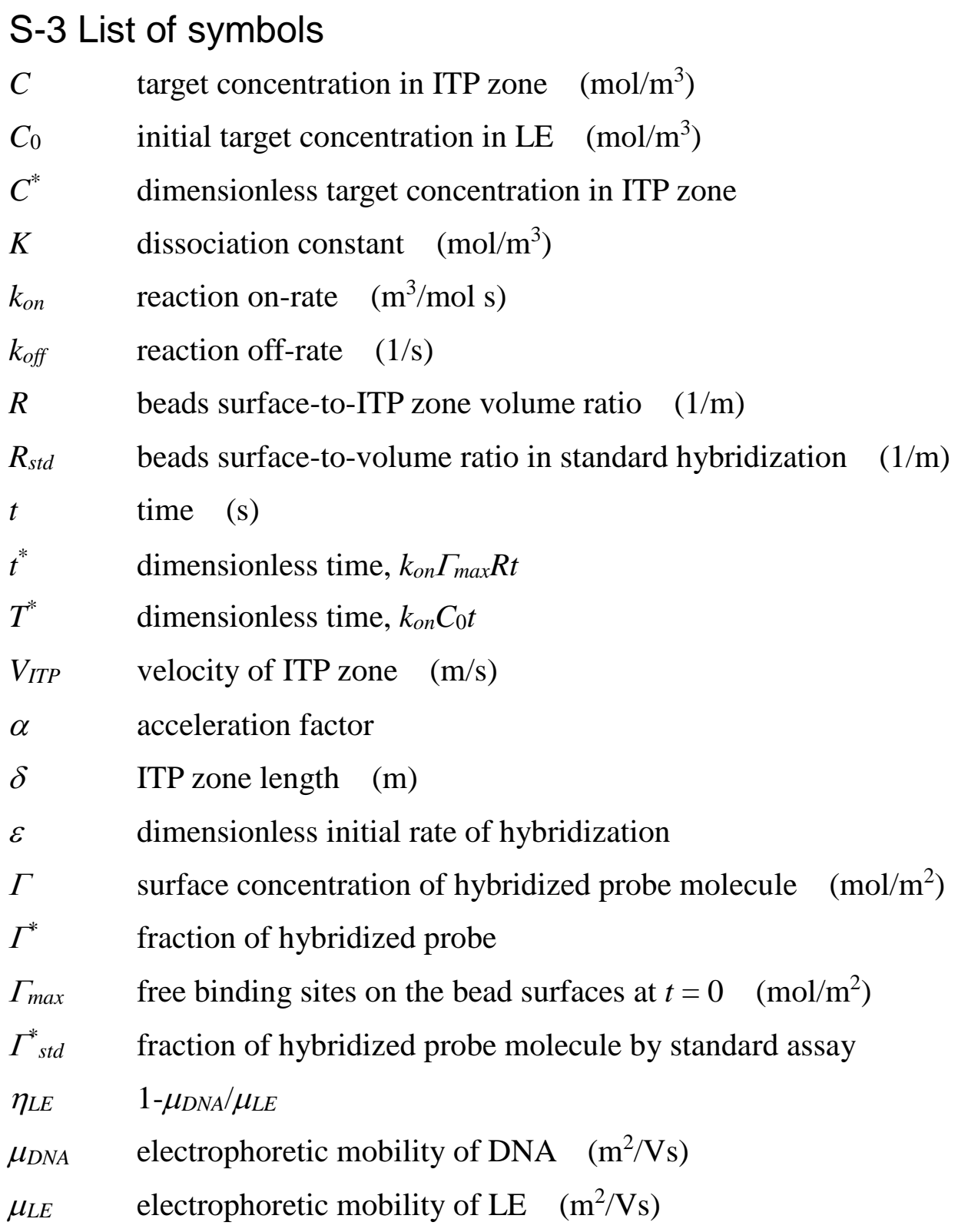

\section{S-4 Approximate analytical model}

We here analyze kinetics of nucleic acid hybridization between suspended targets and immobilized capture probe on the surface of beads to understand the effect of target concentration in the ITP zone. Our analysis offers tools for design and optimization of ITP hybridization experiments.

We model the reaction as second order with off- and on-rate constants, $k_{o f f}$ and $k_{o n}$, respectively, and dissociation constant $K=k_{o f f} / k_{o n}$. Our initial formulation is similar to the model proposed by Bercovici et al. ${ }^{[8]}$ but is here adapted to effectively investigate the fraction of hybridized probes per bead. For a simple analysis, we approximate the concentration of ITP focused targets as uniform with a characteristic zone length of $\delta$ and traveling at a known, constant velocity of $V_{I T P}$. We also assume that the beads distribute uniformly within the ITP zone. The reaction can be expressed as follows: 


$$
\begin{aligned}
& \frac{d \Gamma}{d t}=k_{o n} C\left(\Gamma_{\max }-\Gamma\right)-k_{o f f} \Gamma \\
& \frac{d C}{d t}=\frac{\eta_{L E} V_{I I P}}{\delta} C_{0}-k_{o n} C\left(\Gamma_{\max }-\Gamma\right) R+k_{o f f} \Gamma R,
\end{aligned}
$$

where $C$ and $C_{0}$, respectively, denote volumetric target concentration in the ${ }^{2}$ ITP zone and initial target concentration in the LE with units of $\mathrm{mol} / \mathrm{m}^{3} . \quad \Gamma$ and $\Gamma_{\max }$ respectively denote molar surface concentration of hybrid pairs and free binding sites on the bead surfaces at $t=0$ in units of $\mathrm{mol} / \mathrm{m}^{2}$. The variable $\eta_{L E}$ is defined as, $\eta_{L E}=1-\mu_{D N A} / \mu_{L E}$, where $\mu_{D N A}$ and $\mu_{L E}$ are the electrophoretic mobility of targets and leading anions, respectively. $\quad R$ is the ratio between the total surface area of the beads and the volume of the ITP zone expressed as

$R=n S /(w h \delta)$,

where $n, S, w$, and $h$ are respectively the number of beads, surface area of a single bead, width, and depth of the channel. $\delta$ is the characteristic streamwise (axial) width of the ITP zone.

The governing equations in dimensionless form can be expressed as

$$
\begin{aligned}
& \frac{d I^{*}}{d t^{*}}=\varepsilon\left[\left(1-I^{*}\right) C^{*}-\frac{K}{C_{0}} I^{*}\right], \\
& \frac{d C^{*}}{d t^{*}}=\alpha-C^{*}\left(1-I^{*}\right)+\frac{K}{C_{0}} I^{*},
\end{aligned}
$$

where dimensional variables relate to dimensionless ones as

$$
\begin{aligned}
& t^{*}=t\left(k_{o n} \Gamma_{\max } R\right), \quad I^{*}=\Gamma / \Gamma_{\max }, \quad C^{*}=C / C_{0}, \\
& \alpha=\left(\eta_{L E} V_{I I P} / \delta\right)\left(1 / k_{o n} \Gamma_{\max } R\right), \quad \varepsilon=C_{0} / \Gamma_{\max } R
\end{aligned}
$$

As we discuss in detail later, we term $\alpha$ as the "acceleration factor". $\alpha$ physically represents the ratio of target influx into the ITP zone to the rate of target consumption by hybridization to beads. We further introduce a time scale $T^{*}$ defined as $T^{*}=\varepsilon t^{*}=\left(k_{o n} C_{0}\right) t . \quad \varepsilon$ is a dimensionless initial rate of hybridization. For our experiments, the order of $\varepsilon$ varies from order $10^{-4}$ to unity (for initial target concentrations of $100 \mathrm{fM} 1 \mathrm{nM}$ ). We here will focus on the most relevant and challenging regime of relatively low initial target concentrations where $\varepsilon<<1$, and simplify Eq. (S-4) as follows:

$$
\frac{d I^{*}}{d I^{*}}=\left[\left(1-I^{*}\right) C^{*}-\frac{K}{C_{0}} I^{*}\right] \text {. }
$$

To explore the dynamics of $C^{*}$, we consider the common regime of small hybridized fraction of available binding sites $\Gamma^{*}<<1$, and approximate Eq. (S-5) by 
$\frac{d C^{*}}{d t^{*}}=\alpha-C^{*}$

In this regime, we see Eq.(S-8) is decoupled from Eq.(S-7) and we obtain the analytical solution of $C^{*}$ as

$C^{*}=\alpha\left[1-\exp \left(-t^{*}\right)\right]$

Equation (S-9) indicates that the target concentration in the ITP zone increases and asymptotically approaches the constant value, $\alpha$. Physically, this implies a self-limiting, steady-state balance between target accumulation and reaction. ITP focusing recruits and preconcentrates target into the ITP zone, raising $C^{*}$ in the ITP zone. This has the effect of increasing reaction rate, and this condition continues until the increased reaction rate exactly balances target influx. We here note, Dogan and Bercovici very recently used a numerical approach to show a similar trend. ${ }^{[9]}$ The current analytical model shows explicitly the key parameters and yields a closed-form solution. For example, the time to reach this stable, internal balance of accumulation and reaction is order $1 /\left(k_{\text {on }} \Gamma_{\max } R\right)$. For our experiments, this time is order $50 \mathrm{~s}$. After this, we can assume a constant target concentration as in Eq. (1). Substituting Eq. (1) into Eq. (S-7), we obtain an approximate analytical solution of $\Gamma^{*}$ given by Eq. (2). We provide detailed benchmarking of Eq. (1) and (2) with numerical modeling in S-5.

\section{S-5 Benchmarking approximate analytical solution with numerical model}

We presented an approximate analytical model for hybridization using ITP with two key assumptions: $\varepsilon$ is very small, $\varepsilon<<1$, and negligible $\Gamma^{*}$ for $t^{*}$ of order unity, $\Gamma^{*}<<1$. Here we evaluate the effect of each assumption on the accuracy of the solution by comparing the numerical and approximate analytical models. To obtain the numerical model, we first substituted Eq. (S-1) into Eq. (S-2) as:

$$
\frac{d C}{d t}=\frac{\eta_{L E} V_{I T P}}{\delta} C_{0}-\frac{d \Gamma}{d t} R,
$$

We then integrated Eq. (S-10) with initial conditions of $\Gamma(0)=0$ and $C(0)=0$ as

$$
C=\frac{\eta_{L E} V_{I I P}}{\delta} C_{0} t-I R
$$

Substituting Eq. (S-11) into Eq. (S-1), we obtained

$$
\frac{d \Gamma}{d t}=k_{o n}\left(\frac{\eta_{L E} V_{I I P}}{\delta} C_{0} t-\Gamma R\right)\left(\Gamma_{\max }-\Gamma\right)-k_{o f f} \Gamma \text {. }
$$

We calculated the numerical model of $\Gamma^{*}$ by direct numerical integration of Eq. (S-12). We then calculated $C^{*}$ with Eq. (S-11). 
Figure S4 presents a comparison of the approximate analytical model and numerical model for four initial target concentrations. As a relevant case, we used the experimental conditions, corresponding to an acceleration factor of $\alpha=18.3$, and a scaled total assay time $\left.\left(T^{*} / \varepsilon\right)\right|_{\text {chip }}=25.0$.

First, we focus on the most interesting case of low target concentrations: $100 \mathrm{fM}$ (circle, $\varepsilon=2.72 \times 10^{-5}$ ) and $1 \mathrm{pM}$ (triangle, $\varepsilon=2.72 \times 10^{-4}$ ). As shown in Fig. S4a, the target concentration predicted by the numerical model increases over time $T^{*} / \varepsilon<1$ as target is accumulated in ITP zone. This concentration then reaches a steady state value in time $T^{*} / \varepsilon>1$.

After this initial preconcentration, we see that the analytical model well approximates the steady state associated with a balance between influx and reaction rate, where free target concentration is well characterized by the acceleration factor, $\alpha$ (solid line).

Second, we comment on the (less interesting) high target concentration cases of $10 \mathrm{pM}$ $\left(\varepsilon=2.72 \times 10^{-3}\right)$ and $100 \mathrm{pM}\left(\varepsilon=2.72 \times 10^{-2}\right)$. For initial target concentrations of $100 \mathrm{pM}$, ITP focusing results in initial target influxes well above the reaction rate. Thereafter, the beads become saturated with captured target and newly recruited target causes continual increases in free target concentration. A steady state balance between influx and reaction rate is never really observed for the latter case. For the $10 \mathrm{pM}$ case, the steady state condition is reached quickly and only lasts briefly, and target concentration thereafter increases as the beads are again saturated. In both cases, our approximate analytical model is unable to capture the behavior of $C^{*}$ in the high target concentration regime because of violation of the negligible $\Gamma^{*}$ assumption (i.e., the reaction is limited by bead capacity).

Figure S4b shows fraction of hybridized probes versus the scaled time. We observe that the approximate analytical model (solid lines), Eq. (2), well matches the more complete numerical model for all the initial target concentrations and time scales of order $T^{*} / \varepsilon$ and greater $\left(T^{*} / \varepsilon>1\right)$. For times smaller than about $T^{*} / \varepsilon \square \square$ the model overpredicts $\Gamma^{*}$ since the model assumes that steady state is reached immediately, and that reaction rate immediately matches influx. On the other hand, the numerical model more accurately captures the slowly increasing reaction rate associated with the initial accumulation stages.

We conclude that our approximate analytical model captures target concentration in ITP for (the most interesting) cases of low initial target concentration where the target influx quickly balances with reaction rate and this steady state persists throughout most of the process (as in our experiments). More important to experiment design, the analytical model of fraction of hybridized probes agrees well with the numerical model predictions, particularly for $T^{*} / \varepsilon$ time scale of order unity and greater, and for the full titration range (Fig. S4b and inset). The analytical model therefore helps identify key physical experimental parameters. 

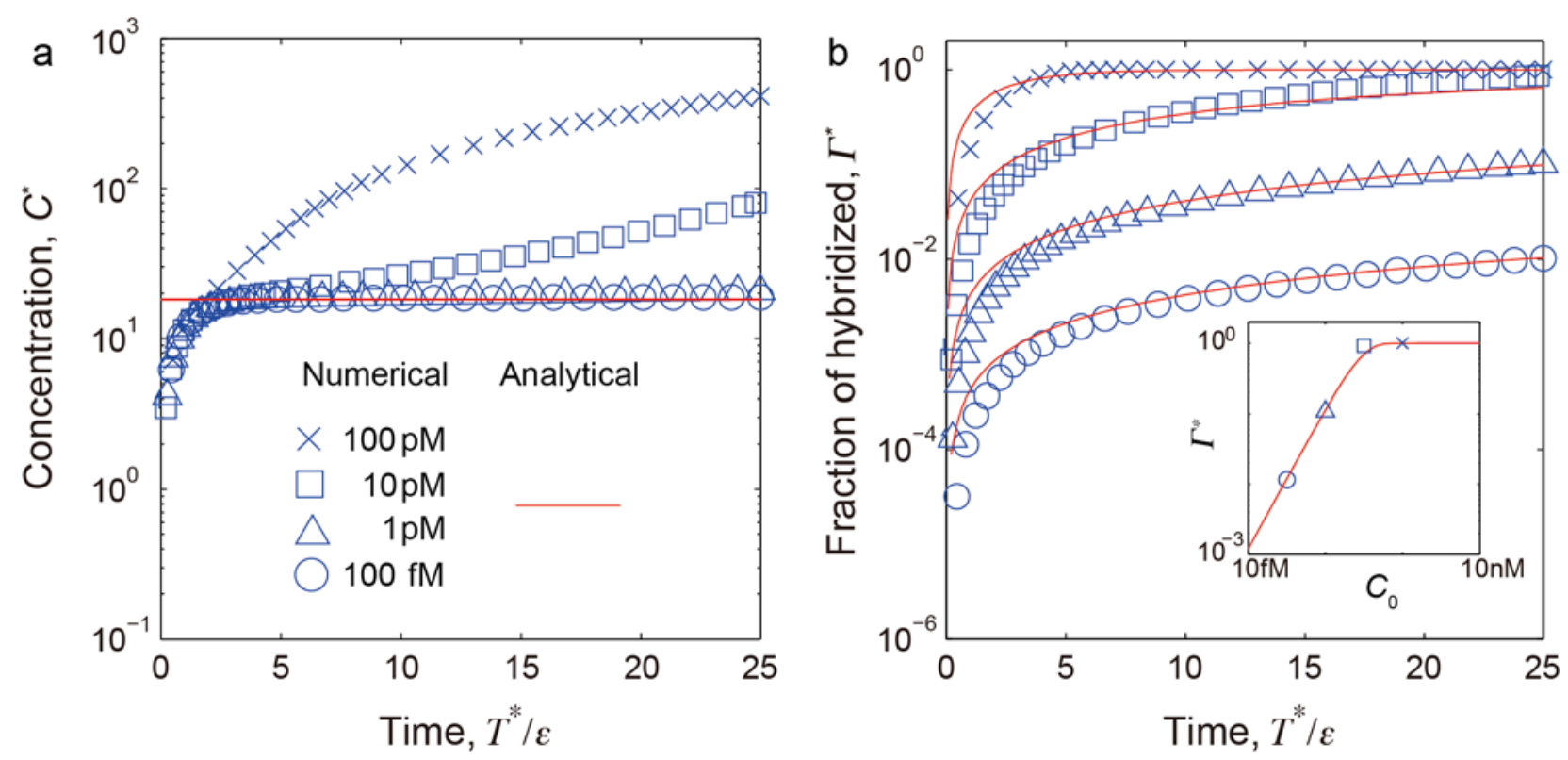

Figure S 4 Comparison of (a) the target concentrations in ITP zone and (b) the fraction of hybridized probes. Plotted are normalized concentration defined as $C / C_{0}$ versus the normalized time scale $T^{*} / \varepsilon$, and fraction of hybridized probes $\Gamma^{*}$ versus the normalized time scale $T^{*} / \varepsilon$. Shown are results for approximate analytical model (solid line) and the numerical model (symbols) at four initial target concentrations. Acceleration factor and scaled total assay time are set as $\alpha=18.3$ and $\left.\left(T^{*} / \mathcal{E}\right)\right|_{\text {chip }}=25.0$, respectively. The inset of B shows the predicted titration curve.

\section{S-6 Theory for standard incubation hybridization and process of estimating dissociation constant and kinetic on-rate}

We here present the following two analytical solutions describing standard incubations: A) an equilibrium solution taking into account target consumption, and B) a transient model assuming a constant target concentration $C_{0}$. Assuming perfect mixing, we use the following governing equations:

$$
\begin{aligned}
& \frac{d \Gamma_{s t d}}{d t}=k_{o n} C\left(\Gamma_{m a x}-\Gamma_{s t l}\right)-k_{o f f} \Gamma_{s t d}, \\
& C=C_{0}-\Gamma_{s t d} R_{s t d},
\end{aligned}
$$

where $R_{\text {std }}$ is the surface-to-volume ratio defined as

$R_{s t d}=n S / V$,

where $n$ is number of beads, $S$ is surface area of a bead, and $V$ is the total volume of the solution.

A) At equilibrium, we obtain the following equation:

$$
0=k_{o n}\left(C_{0}-\Gamma_{e q} R_{s t d}\right)\left(\Gamma_{\operatorname{mox}}-\Gamma_{e q}\right)-k_{\text {off }} \Gamma_{\text {eq }} .
$$


Eq. (S-16) is a quadratic equation in $\Gamma_{e q}$, with a solution

$$
\Gamma_{e q}=\frac{\left(C_{0}+\Gamma_{\max } R_{s t d}+K\right)-\sqrt{\left(C_{0}+\Gamma_{\max } R_{s t d}+K\right)^{2}-4 C_{0} \Gamma_{m a x} R_{s t d}}}{2 R_{s t d}} .
$$

Assuming $\Gamma_{\max } R_{\text {std }} / C_{0}<<1$, we simplify the equilibrium solution under the assumption of constant target concentration as:

$\Gamma_{e q}=\frac{\Gamma_{\operatorname{mox}}}{1+K / C_{0}}$.

B) Under transient conditions, we will assume $C$ maintains a constant value of $C_{0}$. From Eq. (S-13), we obtain a solution

$\Gamma_{\text {std }}=\frac{\Gamma_{\max }}{1+K / C_{0}}\left[1-\exp \left(-k_{o n} C_{0}\left(1+K / C_{0}\right) t\right)\right]$.

At $t \rightarrow \infty$, Eq. (S-19) yields Eq. (S-18).

To obtain estimates for $K$ and $k_{o n}$, we used data obtained in standard incubation cases (and then applied these to predict the ITP dynamics). We first fit data from the standard $20 \mathrm{~h}$ incubation to Eq. (S-17) with a single fitting parameter of $K$ using the 'nlinfit' nonlinear fitting function of MATLAB. We then used this $K$ value and fit data from the standard 30 min incubation to Eq. (S-19) with a single fitting parameter of $k_{o n}$. We also measured the surface concentration of the free binding sites on beads, $\Gamma_{\max }$, as $6 \mathrm{nmol} / \mathrm{m}^{2}$ by measuring the target consumption using a standard incubation and AF488 labeled ssDNA. In Fig. 2 and Fig. S7, we compare predictions from Eqs. (S-17) and (S-19) to the experimental conditions. We also present the final target concentration at equilibrium in the standard incubations. The decrease of the target concentration is significant, especially at low target concentrations, indicating the importance of taking into account the target consumption in our protocol.

\section{S-7 Experimental measurements of ITP parameters}

We here describe our procedure for determining key experimental parameters associated with the ITP process. We used these parameters for our model predictions of ITP-aided focusing and reaction. Our models require estimates of ITP velocity, $V_{I T P}$, and ITP zone length, $\delta$. For simplicity, we assumed a time invariant $\delta$ in our analysis, and used the time-averaged value of $\delta$ resulting in a value of $103 \mu \mathrm{m}$. As our microchannel had seven corners with smaller cross sectional area, the ITP migration velocity was not constant throughout the entire assay. We therefore estimated a representative velocity from the total assay time and the length of microchannel as $V_{\text {ITP }}=670 \pm 30 \mu \mathrm{m} / \mathrm{s}(N=7)$. We obtained mobility of $\mathrm{Cl}^{-}, \mu_{L E}=67 \times 10^{\square}{ }^{9} \mathrm{~m}^{2} /$ Vs using peakmaster (PeakMaster 5.3$)^{[10]}$ and determined that of ssDNA as $\mu_{D N A}=35 \times 10^{\square 9} \mathrm{~m}^{2} / \mathrm{Vs}$ based on a report by 
Stellwagon et al. ${ }^{[1]}$ We stress that these are not meant to be precise estimates but rather engineering estimates useful in assay design and estimation of trends.

\section{S-8 Predicted gains by approximate analytical model in the fraction of hybridized probes using ITP}

The predictions shown in Figures S5a through S5d summarize the fold gains in signal provided by ITP. The solid contour lines represent signal gain defined as the ratio of the fraction of hybridized probes of ITP divided by the fraction hybridized using standard hybridization, i.e. $\Gamma^{*} / \Gamma^{*}$ std. A gain greater than unity represents conditions under which the ITP gives higher fraction of hybridized with the same assay time. For the limiting (and most interesting) case $1<\alpha<<\left(K / C_{0}\right)$, the fraction hybridized by ITP, $\Gamma^{*}$, scales as

$\Gamma^{*}=\alpha /\left(K / C_{0}\right)\left(1-\exp \left(\left(-K / C_{0}\right) T^{*}\right)\right)$

while the standard fraction hybridized, $\Gamma_{\text {std, }}^{*}$, scales as

$\Gamma_{\text {std }}^{*}=1 /\left(K / C_{0}\right)\left(1-\exp \left(\left(-K / C_{0}\right) T^{*}\right)\right)$.

This results in the constant gain $\Gamma^{*} / \Gamma_{\text {std }}^{*}=\alpha$ irrespective of time of the reaction and explains the gain at the low target concentration cases of $100 \mathrm{fM}\left(K / C_{0}=52.8\right.$, Fig. S5a $)$ and $1 \mathrm{pM}\left(K / C_{0}=5.28\right.$, Fig. S5b). For another (least interesting) limiting case, $1>\left(K / C_{0}\right)$, the fraction $\Gamma^{*}$ scales as 1-exp $\left(-\alpha T^{*}\right)$, while $\Gamma^{*}$ std scales as 1-exp(-T*). This results in a gain of $\Gamma^{*} / \Gamma^{*}$ std $=\alpha$ for small $T^{*} / \varepsilon$ and a gain approaching unity for large $T^{*} / \varepsilon$. This in turn explains qualitatively the trends of gain at high concentrations, $10 \mathrm{pM}\left(K / C_{0}=0.528\right.$, Fig. S5c) and $100 \mathrm{pM}\left(K / C_{0}=0.0528\right.$, Fig. S5d). We here note that we developed our approximate analytical model for predicting the fraction of hybridized in $T^{*} / \varepsilon$ time scale greater than unity, and it overestimates the fraction of hybridized in short time scale due to the simplifying assumption of constant target concentration.

We used measured ITP parameters to obtain the model prediction of the acceleration factor $\alpha=18.3$ and non-dimensional assay time $\left.\left(T^{*} / \varepsilon\right)\right|_{\text {chip }}=25.0$ for our experiments (see SI S-7 for detailed measurement of ITP parameters). We show the predictions with a circular symbol in Fig. S5. Shown together with the experimental condition is a dashed line describing the values of $\left.\left(T^{*} / \varepsilon\right)\right|_{\text {chip }}$ and $\alpha$ which are possible given our beads, chip geometry, and ITP chemistry and conditions. The intersection between contour values and this dashed curve describe the possible gains achievable in our chip system. 

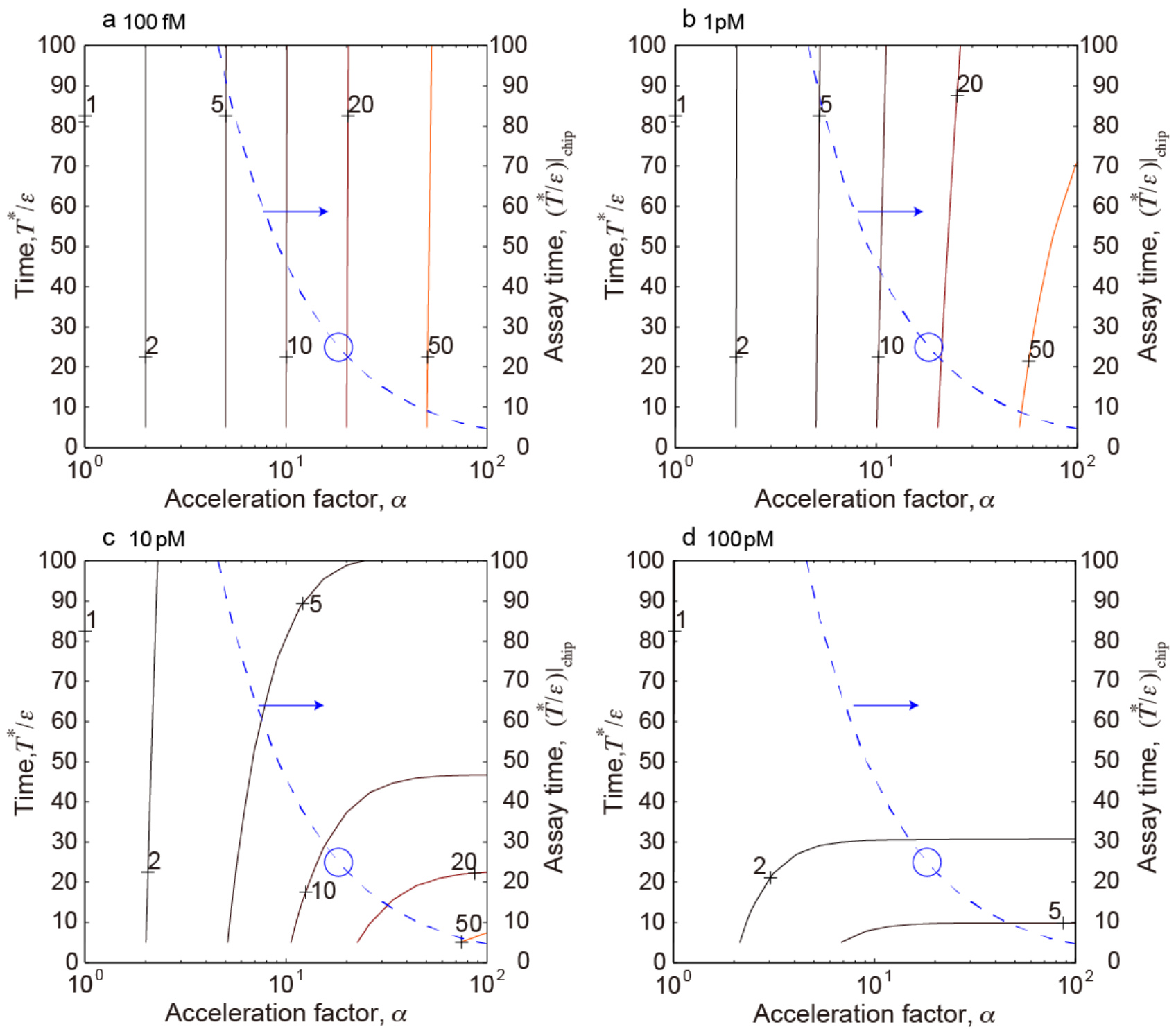

Figure S 5 Effect of ITP focusing on the fraction of hybridized probes for four initial target concentrations: a: $100 \mathrm{fM}$, b: 1 pM, c: 10 pM, and d: 100 pM. Solid contours represent the gain, $\Gamma^{*} / \Gamma_{\text {std }}^{*}$, defined as fraction of hybridized with ITP to that with standard hybridization assuming well-stirred conditions and constant concentrations. The circular symbol represents the model prediction for the experimental condition used in Figs. 2 and $3\left(\alpha=18.3,\left.\left(T^{*} / \varepsilon\right)\right|_{\text {chip }}=25.0\right.$, $k_{\text {on }}=4.27 \times 10^{6}$, and $K=5.28 \times 10^{-12}$ ). The broken line represents of the locus of possible values of $\left.\left(T^{*} / \varepsilon\right)\right|_{\text {chip }}$ versus $\alpha$ for variations associated with only changing the number of beads or electric field.

We also present the fraction of hybridized probes for various acceleration factors by changing the number of beads, keeping other parameters constant in Fig. S6a. Figure S6a is fraction of hybridized probes predicted by the approximate analytical model for a constant channel length and a constant electric field. Higher $\alpha$ corresponds to smaller numbers of beads. The result indicates 
higher $\alpha$ provides higher fraction of hybridized with the same assay time. Figure S6b presents the final fraction of hybridized probes predicted by the approximate analytical model for varying $\alpha$ due to varying electric field, assuming a constant $\delta$. In this case, higher $\alpha$ results in shorter assay times. The results indicate that the final fraction of hybridized probes is insensitive to the electric field strength within the parameter range we explore. This demonstrates that higher electric fields offer shorter assay times without significant reduction in the final fraction of hybridized probes.
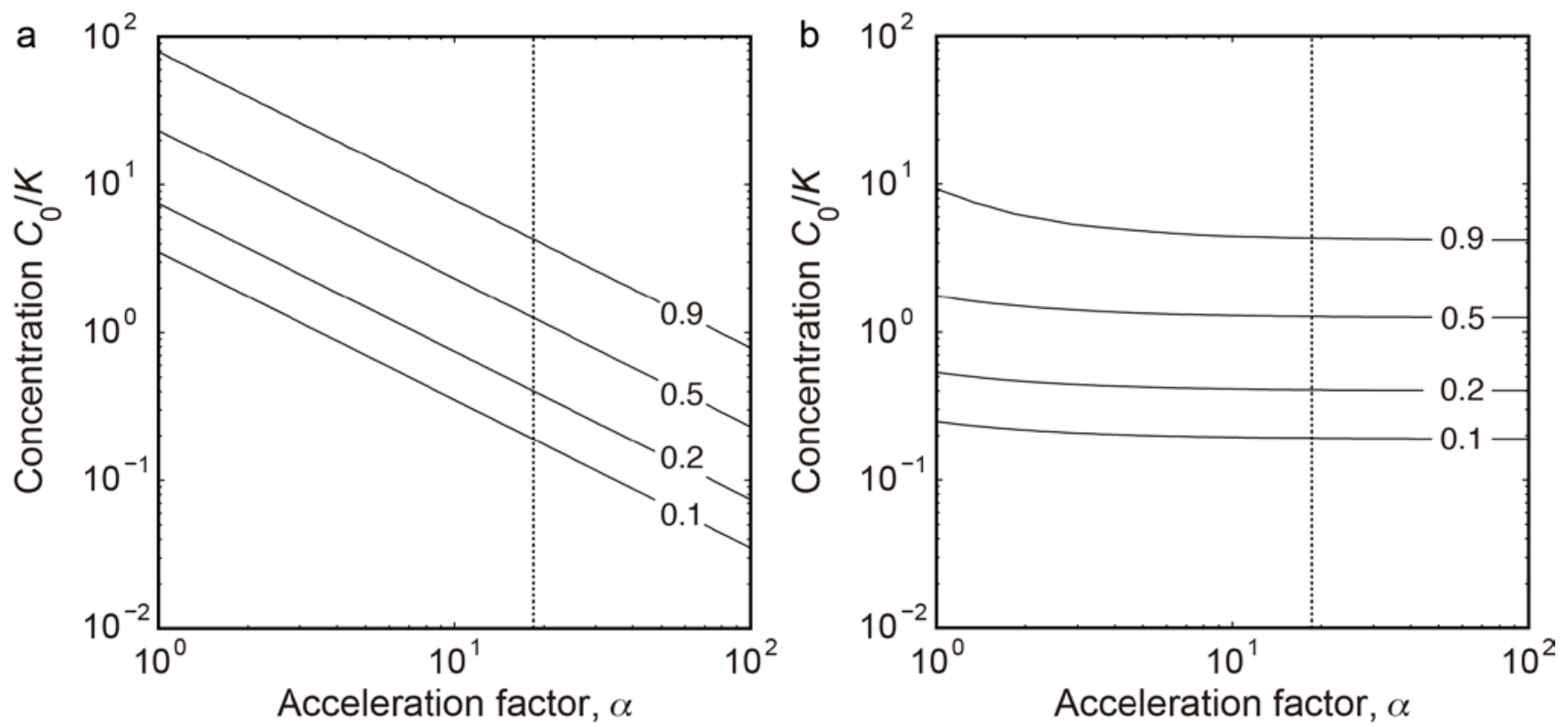

Figure S 6 Final fraction of hybridized from the approximate analytical model for various acceleration factor and initial target concentrations. a): The final fraction of hybridized probes by ITP hybridization with various numbers of beads and/or various cross sectional area. b): The final fraction of hybridized by ITP hybridization at various electric fields under constant channel length and constant $\delta$ assumptions. The dotted lines indicate our experimental conditions.

\section{S-9 Example data from additional titration and specificity experiments}

We here include and discuss additional analyses of the titration and specificity experiments as shown in Figs. 2 and 3 of the main paper. In Fig. S7, we present experimental data on fraction of hybridized probes for ITP (circle) and standard hybridization (squares $20 \mathrm{~h}$ and triangles $30 \mathrm{~min}$ ) for target 9 as compared to target 8 shown in Fig. 2 of the main paper. Shown with the experimental data are approximate analytical model predictions for ITP and standard hybridization. Similar to target 8 , we observe good quantitative model agreement with no fitting parameter. We used the same method to find kinetic parameters and obtained $K=7.27 \times 10^{-12}$ and $k_{\text {on }}=3.65 \times 10^{6}$.

In Fig. S8, we present measurements of specific (ID =9) and nonspecific signal obtained with ITP and standard hybridization. As with target 8, we observed similar specificity in ITP and 
standard $20 \mathrm{~h}$ incubation experiments. Compared to target 8 , target 9 shows higher specificity that reflects the lower melting temperature of the target-probe pair as shown in Table S1. The specificity index in Fig. S8b is the same order of magnitude as target 8.

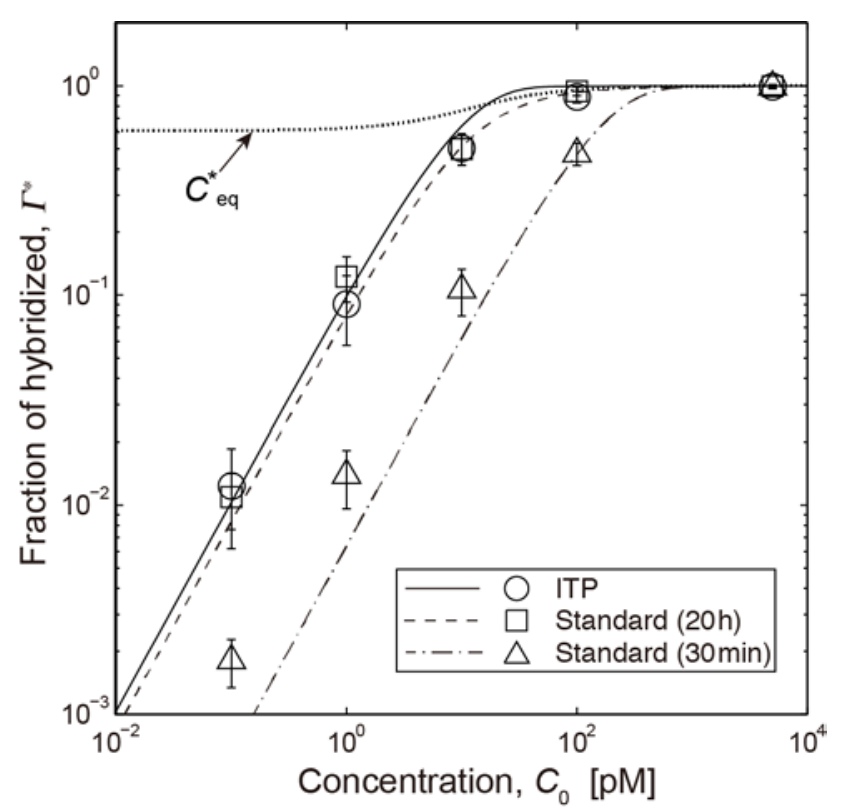

Figure S 7 Experimental data demonstrating quantitative detection of a target molecule using 20 min bead based ITP hybridization and its comparison to well-stirred standard hybridizations performed for $30 \mathrm{~min}$ and $20 \mathrm{~h}$. We obtained the titration curves for target 9 concentraton ranging from $100 \mathrm{fM}$ to $5 \mathrm{nM}$. Along with experimental data (symbols), we show prediction of the analytical model for ITP hybridization (solid) with no fitting parameter. We also show results of analytical models for the standard $20 \mathrm{~h}$ (broken) and $30 \mathrm{~min}$ (dashed-dotted) hybridizations. The range bars in the figure indicate the standard deviation across repetitiation ( $N=6$ for standard or $N=9$ for ITP). The dotted line shows the ideal equilibrium state target concentration at standard hybridization conditions. 
a
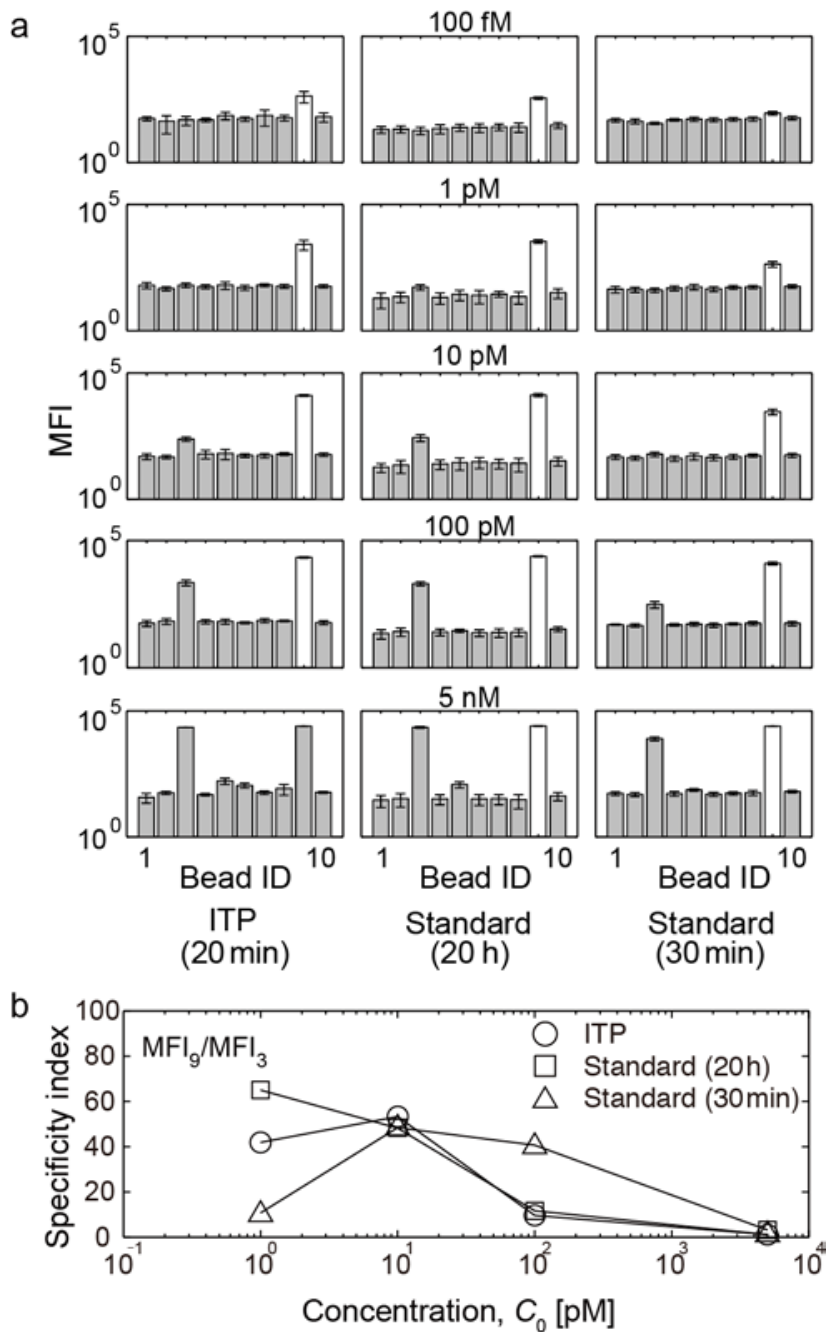

Figure S 8 Comparison of specific and nonspecific signal between ITP hybridization and standard incubation (at both $20 \mathrm{~h}$ and $30 \mathrm{~min}$ ). a): Averaged MFI (log scale) for target 9 hybridized with matching probe (bead ID=9) and nine beads types with non-matching probes. The range bars in the figure indicate the standard deviation across $N=3$ for standard cases and $N=5$ for ITP. b): Specificity index for target 9 (ratio of averaged MFI between specific binding and the highest non-specific binding (bead ID=3).

\section{References}

[1] a) L. A. Marshall, A. Rogacs, C. D. Meinhart, J. G. Santiago, J Chromatogr A 2014, 1331, 139-142; b) J. I. Molho, A. E. Herr, B. P. Mosier, J. G. Santiago, T. W. Kenny, R. A. Brennen, G. B. Gordon, B. Mohammadi, Anal Chem 2001, 73, 1350-1360.

[2] a) T. K. Khurana, J. G. Santiago, Anal Chem 2008, 80, 6300-6307; b) G. Garcia-Schwarz, A. Rogacs, S. S. Bahga, J. G. Santiago, J Vis Exp 2012, e3890.

[3] G. Garcia-Schwarz, M. Bercovici, L. A. Marshall, J. G. Santiago, J Fluid Mech 2011, 679, 455-475.

[4] A. Persat, J. G. Santiago, New J Phys 2009, 11, 075026. 
[5] J. S. Paschkewitz, J. I. Molho, H. Xu, R. Bharadwaj, C. C. Park, Electrophoresis 2007, 28, 4561-4571.

[6] a) J. G. Santiago, S. T. Wereley, C. D. Meinhart, D. J. Beebe, R. J. Adrian, Exp Fluids 1998, 25, 316-319; b) C. D. Meinhart, S. T. Wereley, J. G. Santiago, Exp Fluids 1999, 27, 414-419.

[7] C. D. Meinhart, S. T. Wereley, J. G. Santiago, J Fluids Eng 2000, 122, 285-289.

[8] M. Bercovici, C. M. Han, J. C. Liao, J. G. Santiago, Proc Natl Acad Sci USA 2012, 109, 11127-11132.

[9] O. Dagan, M. Bercovici, Anal Chem 2014.

[10] M. Jaros, K. Vcelakova, I. Zuskova, B. Gas, Electrophoresis 2002, 23, 2667-2677.

[11] N. C. Stellwagen, C. Gelfi, P. G. Righetti, Biopolymers 1997, 42, 687-703. 